Investigators

This study was done by university researchers under contract to NIOSH. The primary researchers were Gary M. Marsh, PhD, University of Pittsburgh, and Nurtan A. Esmen, PhD, University of Illinois at Chicago.

Why did NIOSH do the study?
The union that represented some workers at the smelter asked NIOSH to study worker health. The union and people in the nearby community wanted to know if health problems were related to working in or living near the smelter. Although this study only included smelter workers, NIOSH thought the results would help answer questions about health in the community. Many people in the community worked at the smelter. Also, smelter operators had data about exposures in the plant. These data would help researchers better understand whether smelter-related exposures were linked to health problems. Because a study of the smelter workers had been done in the 1980s, this study was possible.
What were the goals of the study?
The researchers set the following four goals for the study.

Compare death rates and causes of death in smelter workers with those in the general population.

$\checkmark$ Describe the work environment in the smelter by work area, job title, and levels of exposure to six agents.

$\checkmark$ Describe the smoking history of smelter workers and use this information to help understand the cause-of-death results.

$\checkmark$ Examine whether specific causes of death were related to exposures in the smelter work setting.
Who was included in the study?
The study included 2,422 men who worked in the smelter, mill, or sulfur plant for 3 or more years between January 1946 and April 1996. For the years 1949 through 2000, the researchers found out whether these workers were living or deceased. If the workers were deceased, the researchers obtained information about their cause of death. 


PAGE 2
What information
did the researchers
use?

What information

The researchers used information from national and state records to learn whether people in the study had died. The researchers then obtained information about the causes of those deaths. They also used information from records in the smelter about the age, race, and sex of people in the study. They compared the number of deaths of smelter workers to the number that would be expected in groups of people who did not work in the smelter. These included the general population of the United States and the population of the counties around the smelter. They made these comparisons for all causes of death combined, for groups of diseases (such as all cancer or all lung disease), and for specific diseases (such as lung cancer or cerebrovascular disease, also known as stroke). They used statistical tests to decide if the results were meaningful. When they saw meaningful differences, they looked to see if death rates were related to exposure. They got exposure information from smelter records and by interviewing workers about smelter operations. To look at exposure, they grouped people by how long they worked in the plant, what department they worked in, their job title, and the level of exposure. They estimated exposure levels for lead, arsenic, cadmium, cobalt, dust, and sulfur dioxide based on records of workplace air sampling done by smelter operators.
What did the researchers find?
All causes of death. Of the workers in the study, 961 (41\%) were deceased. Researchers found a cause of death for 878 of these. The total number of deaths expected among smelter workers was based on national and local county rates. For all causes of death, all types of cancer, all types of heart disease, respiratory disease other than cancer, and many of the specific causes of death, the rates in the surrounding counties were similar to the national rates.

Cancer deaths. Of the workers in the study, 228 died of cancer. This was $18 \%$ lower than expected based on national rates and 16\% lower based on local county rates. When specific types of cancer were looked at separately, some differences between smelter workers and the general population were found. Most of these differences were based on numbers of deaths so small they were not thought to be important. Eleven workers died of cancer of the central nervous system, an excess of 39\% compared to the general population. By statistical tests, this difference was not meaningful.

Noncancer deaths. The results did not show that smelter workers had a meaningful increase in the risk of death from any other cause of death.

Levels of exposure. The average exposure levels for lead and sulfur dioxide were close to the current workplace limits for these agents. For arsenic, cadmium, cobalt, and dust, the past average exposure levels were much lower than current limits. 


\begin{tabular}{ll}
\hline PAGE 3 & \multicolumn{1}{c}{ COPPERHILL SMELTER WORKER STUDY } \\
\hline researchers find? & Causes of death and exposure. After examining all causes of death and all \\
(continued) & measures of exposure, the researchers looked at two findings more closely. \\
& These were the link between arsenic exposure and stroke and the link \\
between cadmium exposure and bronchitis. & \\
& Stroke and arsenic exposure. Seventy workers died of stroke. The risk of death \\
& from stroke was higher for people who worked longer and for people with \\
& more arsenic exposure. By statistical tests, this difference was not \\
& meaningful. For example, workers with the highest level of total exposure \\
& were 1.5 times more likely to die of stroke than unexposed workers, but the \\
& p value, a measure of significance, was 0.17 ; a p value of 0.05 or less is \\
& regarded as statistically significant. \\
& Bronchitis and cadmium exposure. Seven workers died of bronchitis. The risk \\
& of bronchitis death was higher for some exposed workers than for \\
& unexposed workers. This finding, however, was not the same for all \\
& exposure groups or measures. For example, workers with a moderate level of \\
& total cadmium exposure were 14.8 times more likely to die of bronchitis \\
& than unexposed workers. But, workers with the highest level of exposure \\
& were only 3.8 times more likely to die of bronchitis. The $p$ value for these \\
& findings was 0.06, not statistically significant.
\end{tabular}

Are there any limitations to this study?
As with most studies of this type, some factors make it hard for researchers to draw firm conclusions about the findings. First, because complete and accurate information about cigarette smoking was not available, the researchers could not investigate how cigarette smoking affected causes of death. But, it is unlikely that this limitation had an important effect on the conclusions. Second, this study was smaller than studies of other workplaces. The small size makes it less likely that the findings will be statistically significant, even if there is a real risk. Because of this, it is helpful to look at the how the findings of this study fit in with the findings of other studies of smelter workers or of other workers with similar exposures.

\section{What do the} results mean?
Death rates for Copperhill smelter workers were lower than expected for all causes of death and from specific cancer and noncancer causes. This is not an unusual finding in studies of workers. So, researchers looked to see if workplace exposures were related to the risk of death from specific causes. One finding of interest was for arsenic and stroke. Another was for cadmium and bronchitis. The researchers concluded it is unlikely that arsenic exposure caused increased stroke risk or that cadmium exposure caused increased bronchitis disease risk in Copperhill workers. 
What do the results This conclusion is based on the detailed analyses of the findings from this mean? study and on evidence from other studies about these exposures and diseases. It is also important to note that other studies of smelter workers have shown that arsenic exposure is related to respiratory cancer. Arsenic exposures in the Copperhill smelter were lower than in other smelters studied. The researchers in this study did not see an increase in respiratory cancer risk.

National Institute for Occupational Safety and Health (NIOSH)

Division of Surveillance, Hazard Evaluations and Field Studies (DSHEFS)

Hazard Evaluations and Technical Assistance Branch (HETAB)

NIOSH-HETAB MS R-9

4676 Columbia Parkway

Cincinnati, $\mathrm{OH} 45226$

To request a copy of the 103-page technical report, call:

\section{1-513-841-4382}

For more information about the NIOSH Health Hazard Evaluation Program, visit on-line at: http://www.cdc.gov/niosh/hhe 